\title{
Diffuse Large B-cell Lymphoma with Massive Portal Vein Tumor Thrombosis in a Patient with Alcoholic Cirrhosis: A Case Report and Literature Review
}

\author{
Mitsuteru Natsuizaka ${ }^{1,3}$, Mineo Kudo ${ }^{1}$, Mio Suzuki ${ }^{1}$, Masatoshi Takano ${ }^{1}$, \\ Masako Tsuyuguchi ${ }^{1}$, Naoyuki Kawamura ${ }^{1}$, Shinsuke Noguchi ${ }^{2}$, Akinori Wada ${ }^{2}$, \\ Masanobu Nakata ${ }^{2}$, Masahiro Ogasawara ${ }^{2}$, Yoshio Kiyama ${ }^{2}$, \\ Masahiro Asaka ${ }^{3}$ and Masaharu Kasai ${ }^{2}$
}

\begin{abstract}
We describe a case of diffuse large B-cell lymphoma with massive portal vein tumor thrombosis in a patient with alcoholic cirrhosis. The tumor was detected only in the intrahepatic portal vein and the spermatic cord by FDG-PET/CT. Percutaneous liver biopsy and orchiectomy were performed and histological examination revealed diffuse large B-cell lymphoma. The tumor showed complete response after six courses of the combination chemotherapy. Portal vein tumor thrombosis of malignant lymphoma is extremely rare; moreover, it is possible that this is the first case of malignant lymphoma originating from the spermatic cord producing portal vein tumor thrombosis.
\end{abstract}

Key words: primary malignant lymphoma of spermatic cord, portal vein tumor thrombosis, diffuse large Bcell lymphoma, liver cirrhosis, FDG-PET, hepatocellular carcinoma

(Inter Med 48: 805-808, 2009)

(DOI: 10.2169/internalmedicine.48.1648)

\section{Introduction}

Hepatocellular carcinoma (HCC) occasionally develops portal vein tumor thrombosis (PVTT). PVTT can also result from pancreatic cancer, renal cell carcinoma and cholangiocarcinoma (1). Concerning the hepatosplenic lesions of malignant lymphoma, a tumor mass or disseminated lymphoma in the liver or spleen is sometimes encountered, but PVTT of malignant lymphoma is very rare. To the best of our knowledge, only a few cases of malignant lymphoma with PVTT have been reported thus far (2-5). We present a case of alcoholic liver cirrhosis diagnosed with diffuse large Bcell lymphoma (DLBCL) with massive PVTT.

\section{Case Report}

A 75-year-old man was admitted to our hospital in 2004 because of bleeding from the esophageal varices, and was treated with endoscopic variceal injection sclerotherapy. He was diagnosed with alcoholic liver cirrhosis as well. CT and ultrasonography were performed every 3 to 6 months after discharge. In 2007, periodic CT examination detected massive portal vein thrombosis (Fig. 1a). The portal vein thrombus largely extended to the bilateral hepatic lobes. No tumor thrombus was observed in other portal venous systems including the main portal vein trunk. However no spaceoccupying lesion indicating the primary site of hepatocellular carcinoma (HCC) was detected, HCC was strongly suspected because of the massive PVTT and the patient's history of alcoholic liver cirrhosis. On the other hand, the tu-

\footnotetext{
${ }^{1}$ Department of Gastroenterology, Sapporo Hokuyu Hospital, Sapporo, ${ }^{2}$ Department of Hematology, Sapporo Hokuyu Hospital, Sapporo and ${ }^{3}$ Department of Gastroenterology and Hematology, Hokkaido University Graduate School of Medicine, Sapporo Received for publication August 30, 2008; Accepted for publication January 25, 2009 Correspondence to Dr. Mitsuteru Natsuizaka, tosho@hokuyu-aoth.org
} 

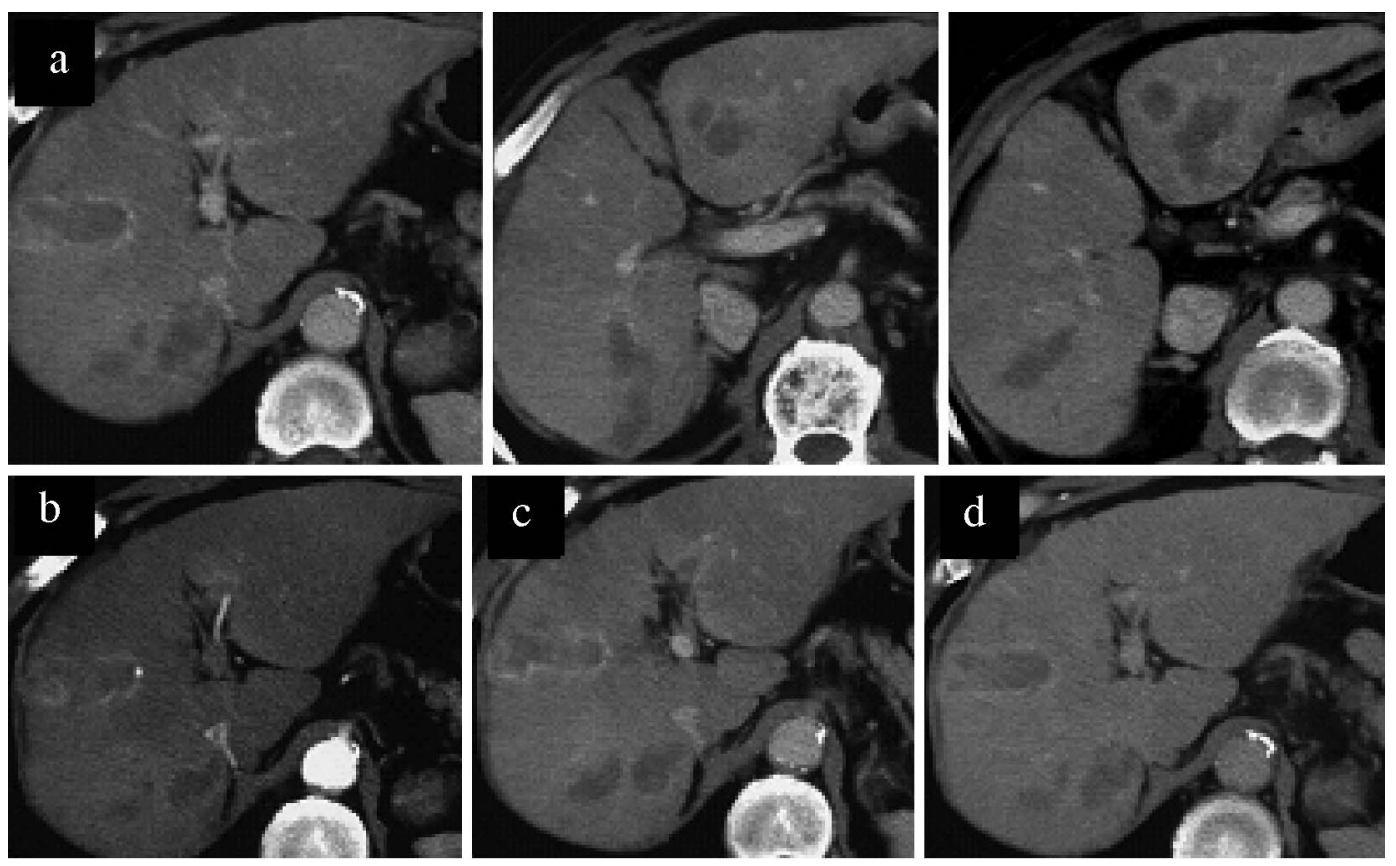

Figure 1. (a) CT showed massive intrahepatic portal vein tumor thrombosis (Images in portal phase of 3 phase dynamic CT). (b, c, d) The tumor did not show hyperenhancement in arterial phase of 3 phase dynamic CT. a: arterial phase, b: portal phase, c: late venous phase.
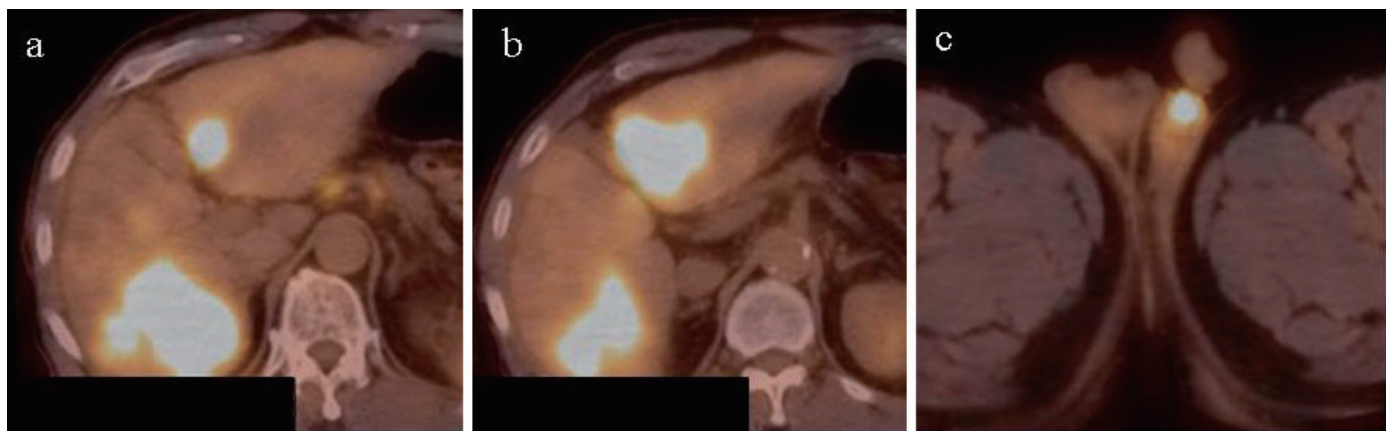

Figure 2. FDG-PET/CT showed marked accumulation in the portal vein tumor thrombosis and a small abnormal uptake in the scrotum. No other significant uptake was detected in other organs and lymph nodes.

mor was a hypovascular tumor and did not show hyperenhancement in the early phase of 3 phase dynamic CT scanning (Figs. 1b, 1c, 1d), and tumor markers of HCC such as alpha-fetoprotein and protein induced by the absence of vitamin $\mathrm{K}$ or by antagonist II were all within normal range. Malignant lymphoma was also suspected because of elevated levels of serum lactate dehydrogenase (LDH) and serum interleukin 2 receptor (sIL-2R). PET/CT showed marked accumulation in the intrahepatic PVTT and weak accumulation in the scrotum (Fig. 2). No significant lymph node swelling was detected by CT and PET/CT except for the above lesions. The left testis and spermatic cord were surgically resected to obtain a definitive diagnosis. A few small nodules were identified only in the spermatic cord but not in the testis; histological examination revealed nonHodgkin's lymphoma (Fig. 3a). The lymphoma cells showed positive immunohistochemical staining for CD20, L26, and
MUM-1 and negative staining for CD3, CD5, CD10, and bcl-6. Based on these findings, the histological diagnosis was diffuse large B-cell lymphoma (DLBCL). Percutaneous liver biopsy was also performed to obtain definite diagnosis of PVTT. The histological examination of the PVTT revealed DLBCL histologically similar to the lesion of the left spermatic cord (Fig. 3b). Bone marrow aspiration showed no involvement of lymphoma cells, and he was diagnosed with DLBCL with massive intrahepatic portal vein tumor thrombosis. After six courses of combination chemotherapy, including cyclophosphamide, doxorubicin, vincristine, prednisolone, and rituximab (R-CHOP), CT and ultrasonography of the patient showed complete response of the tumor (Fig. 4), which was maintained for 16 months after completion of the combination chemotherapy. 

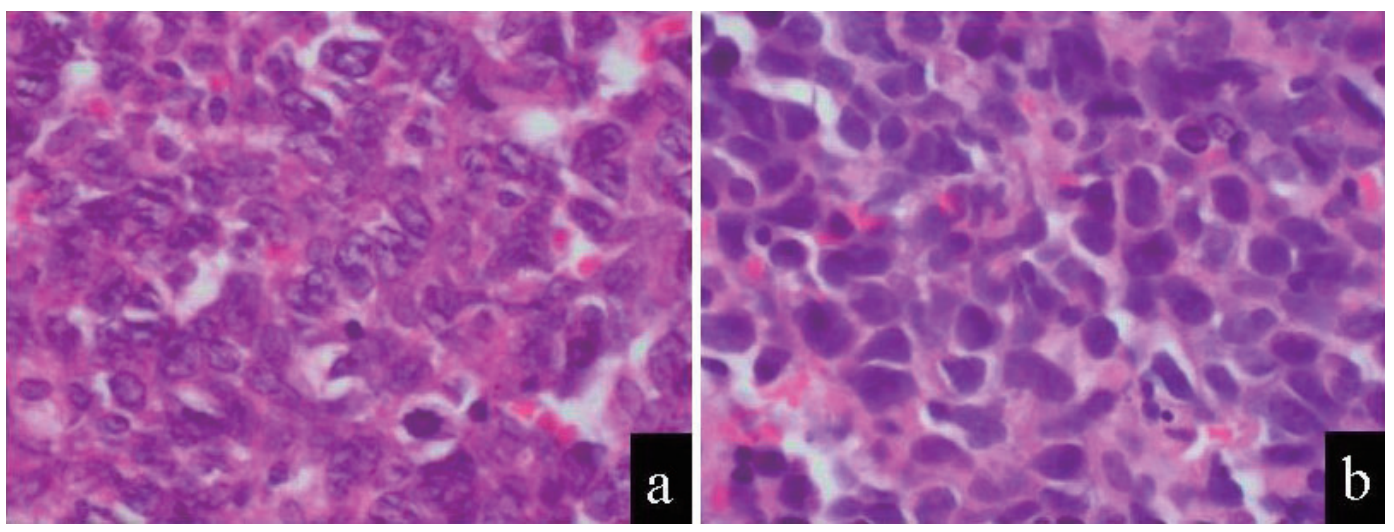

Figure 3. (a) Surgical resection of spermatic cord revealed DLBCL (Hematoxylin and Eosin staining, original magnification $\times 400$ ). (b) Percutaneous biopsy of PVTT also revealed DLBCL histologically similar to the lesion of the left spermatic cord (Hematoxylin and Eosin staining, original magnification $\times 400)$.
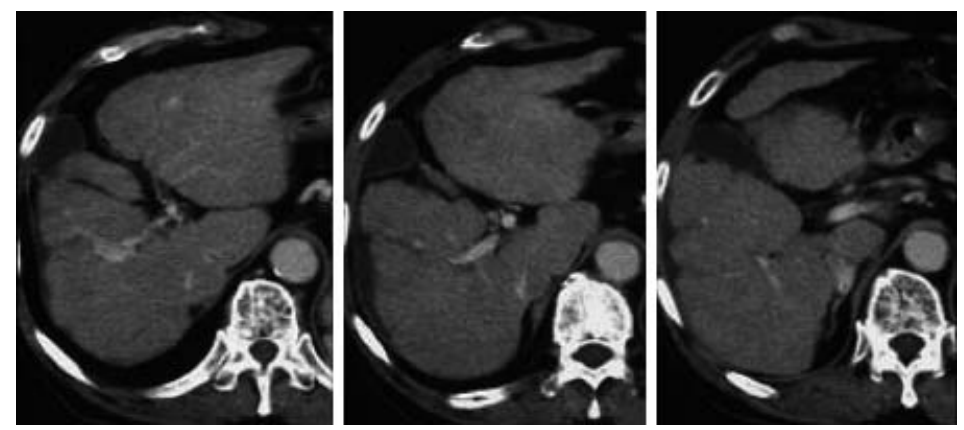

Figure 4. After receiving six courses of the combined chemotherapy, the patient achieved complete response of the tumor and recovered normal blood flow of the portal vein.

Table 1. Literature Review of Malignant Lymphoma with PVTT

\begin{tabular}{cccccccc}
\hline case [Ref] & age & sex & histology & primary site & therapy & response & follow-up period \\
\hline $1[2]$ & 71 & male & B-cell lymphoma & liver & SR & NA & NA \\
$2[3]$ & 55 & female & DLBCL & liver & SR and CT & CR & 4.5 years \\
$3[4]$ & 62 & female & DLBCL & mesenchyma & CT & CR & 7.5 years \\
$4[5]$ & 55 & male & DLBCL & spleen & CT & CR & 6 years \\
present case & 78 & male & DLBCL & spermatic cord & CT & CR & 16 months \\
\hline
\end{tabular}

Abbreviations, SR: surgical resection, CT: chemotherapy, NA: not available, CR: complete response

\section{Discussion}

In the present case, periodic CT imaging study revealed massive portal vein thrombosis, raising the suspicion of HCC secondary to advanced alcoholic liver cirrhosis. However, the tumor did not show hyperenhancement in the early phase of dynamic CT and tumor markers of HCC were all within normal range and space-occupying lesions were not detected in the liver. Furthermore, PET/CT showed marked accumulation with standardized radioactivity uptake value $>$ 15 at the portal vein thrombosis of the liver. Although some reports have indicated that the detection rate of FDG-PET for HCC is high (6), the most common view is that the sensitivity of FDG-PET for HCC is around 50\%, which is not sufficient $(7,8)$. FDG-PET does not usually show a strong accumulation in HCC cases. On the other hand, the detection rate of FDG-PET for malignant lymphoma, especially DLBCL, is $>90 \%$, and FDG-PET shows strong accumulation in DLBCL cases (9). Currently, FDG-PET is one of the most necessary modalities to diagnose malignant lymphoma. Our result of PET/CT showed the possibility of malignant lymphoma, therefore we performed the histological examinations, percutaneous liver biopsy and orchiectomy. PET/CT was a very useful modality, which led us to the correct diagnosis in this case.

Portal vein tumor thrombosis of malignant lymphoma is an extremely rare entity except in patients receiving orthotopic liver transplantation (10). Irie et al reported the first case in 1996 (2), and to the best of our knowledge, only 
four cases have been reported thus far (Table 1) (2-5). The primary sites were the liver in two cases and the mesenchyma and spleen in the other two cases. It is also noteworthy that all reported cases of malignant lymphoma developing PVTT were B-cell lineage lymphoma, most commonly DLBCL. In the previous report, one case of malignant lymphoma with PVTT underwent surgical resection (2). Two cases received combined chemotherapies $(4,5)$, and one case underwent both surgical resection and combined chemotherapy (3). No recurrence was observed during 4.5 to 6 years of follow-up after therapy in the three reported cases. No recurrence was observed for 16 months after chemotherapy in our case as well. PVTT of malignant lymphoma does not seem to convey poor prognosis, and appropriate therapy should be initiated in such cases.

It is interesting that lymphoma cells were detected only in a small lesion of the spermatic cord, while a massive accumulation of lymphoma cells were present in the intrahepatic portal vein without a liver mass in the present case. There is a possibility that this is a case of primary hepatic lymphoma (PHL) with PVTT; however, it is very unusual for PHL to metastasize solely onto the spermatic cord. Based on the absence of space-occupying lesions in the liver and the absence of lymph node swelling, it is possible that our case is the first case of malignant lymphoma originating from the spermatic cord producing massive PVTT. Male gonadal lymphoma is also a rare entity. Of these lymphomas, testicu- lar lymphoma is the most frequent and accounts for 5 to $10 \%$ of testicular neoplasms and 1 to $2 \%$ of all nonHodgkin's lymphomas (11-13). Primary lymphoma of the spermatic cord is extremely rare and only a small number of cases have been reported to date $(11,12)$. However, the clinicopathological features of primary lymphoma of the spermatic cord remain to be determined because of its extremely low occurrence, it appears to have similar biological behavior as primary testicular lymphoma $(6,7)$. Both have a propensity to spread aggressively to extranodal sites such as central nervous system, skin, pleura, lung, and Waldeyer's ring (11-13). Two cases of primary lymphoma of the spermatic cord metastasizing to the organs related to portal venous system have also been reported; one metastasized to the liver and the other showed intravascular lymphomatosis and metastasized to the spleen $(14,15)$. The lymphoma cells might metastasize to the intrahepatic portal vein from the spermatic cord because of its ability of aggressive spread and invasion in our case, however the detailed mechanism remains to be elucidated.

In summary, we present the rare case of DLBCL with PVTT. It is possible that our case is the first reported case of primary lymphoma of the spermatic cord developing massive PVTT. Malignant lymphoma with PVTT is extremely rare, but we should consider that non-Hodgkin lymphoma, especially DLBCL, may sometimes develop PVTT.

\section{References}

1. Laissy JP, Trillaud H, Douek P. MR angiography: noninvasive vascular imaging of the abdomen. Abdom Imaging 27: 488-506, 2002.

2. Irie H, Honda H, Kaneko K, et al. Primary malignant lymphoma in the porta hepatis: a case report. Abdom Imaging 21: 448-450, 1996.

3. Yoneyama F, Nimura Y, Kamiya J, et al. Primary lymphoma of the liver with bile duct invasion and tumoral occlusion of the portal vein: report of a case. J Hepatol 29: 485-488, 1998.

4. Matsumoto S, Mori H, Takaki H, Ishitobi F, Shuto R, Yokoyama S. Malignant lymphoma with tumor thrombus in the portal venous system. Abdom Imaging 29: 460-462, 2004.

5. Kanemura N, Tsurumi H, Yamada T, Kojima Y, Kasahara S, Moriwaki H. Diffuse large B-cell lymphoma with tumor thrombosis in the portal vein. Int J Hematol 84: 282-283, 2006.

6. Sugiyama M, Sakahara H, Torizuka T, et al. 18F-FDG PET in the detection of extrahepatic metastases from hepatocellular carcinoma. J Gastroenterol 39: 961-968, 2004.

7. Khan MA, Combs CS, Brunt EM, Lowe VJ, Wolverson MK, Solomon H, et al. Positron emission tomography scanning in the evaluation of hepatocellular carcinoma. J Hepatol 32: 792-797, 2000 .
8. Jeng LB, Changlai SP, Shen YY, Lin CC, Tsai CH, Kao CH. Limited value of $18 \mathrm{~F}$-2-deoxyglucose positron emission tomography to detect hepatocellular carcinoma in hepatitis B virus carriers. Hepatogastroenterology 50: 2154-2156, 2003.

9. Burton C, Ell P, Linch D. The role of PET imaging in lymphoma. Br J Haematol 126: 772-784, 2004.

10. Moody AR, Wilson SR, Greig PD. Non-Hodgkin lymphoma in the porta hepatis after orthotopic liver transplantation: sonographic findings. Radiology 182: 867-870, 1992.

11. Vega F, Medeiros LJ, Abruzzo LV. Primary paratesticular lymphoma: a report of 2 cases and review of literature. Arch Pathol Lab Med 125: 428-432, 2001.

12. Okabe M, Kurosawa M, Suzuki S, et al. Primary lymphoma of spermatic cord. Leuk Lymphoma 40: 663-666, 2001.

13. Vitolo U, Ferreri AJ, Zucca E. Primary testicular lymphoma. Crit Rev Oncol Hematol 65: 183-189, 2008.

14. Hautzer NW, Nikolai V. Primary lymphoma of the spermatic cord. Br J Urol 58: 565-566, 1986.

15. Au WY, Shek WH, Nicholls J, Tse KM, Todd D, Kwong YL. Tcell intravascular lymphomatosis (angiotropic large cell lymphoma): association with Epstein-Barr viral infection. Histopathology 31: 563-567, 1997.

(C) 2009 The Japanese Society of Internal Medicine http://www.naika.or.jp/imindex.html 